\title{
Interactive comment on "Validation, comparison, and integration of GOCI, AHI, MODIS, MISR, and VIIRS aerosol optical depth over East Asia during the 2016 KORUS-AQ campaign” by Myungje Choi et al.
}

\section{Anonymous Referee \#2}

Received and published: 28 May 2019

The study validates and compares aerosol property, aerosol optical depth (AOD), of several satellites both GEO and LEO with AERONET or each satellite. The paper shows AOD accuracy of both GEO and LEO and indicates why the bias difference occurs. This study is useful to know what bias they have, to improve the retrieval algorithms and to select AOD data for air quality models. Below are my comments for the authors to consider before publishing the paper:

1. The paper mentions about using satellite combined AOD for air-quality model and mentions that an observation campaign in the paper also lead to improve air quality 
model. Please added some sentences what problem of present models has (only for high time resolution?). Why combined AOD using GEO and LEO is useful (than that using only GEO)? If possible, please tell me the condition of data selection to make combine data because the authors validate and compared several GEO and LEO satellite data and also mention their bias. 2. In the abstract, the author has said that cloud screening is AOD difference between sensors. However, it is difficult to understand it from the paper. Please tell me why you did not meet the condition of each cloud screening when you compare AODs from several satellite. 3. Please add line between wavelength and the vertical line of MISR in Figure $1 \mathrm{~b}$ (or remove lines between points). Please explain why MISR AOD accuracy is not good when MISR AOD is large over Land if you have some opinion. Please modify the figure because error bar is not clear. 4. Page 9, line17: "highly accurate", this expression is ambiguous. Please add what accuracy AODs are. In Page9: Does GOCI use Cox and Monk method over ocean? The AOD result of GOCI over ocean has positive bias, but in sentence the author have said "negative bias". Is it correct? 5. Figure 3. Please explain why MODIS DT and DB overestimate AOD. 6. Page10, line15 : "high accuracy", this expression is ambiguous. Please explain why accurate it is, and why GEO results have continuous spatiotemporal distribution. 7. Figure 4, 5: I think that it is good to add some discussion including the wind speed. 8. Please correct to correct one. Page8 linr8-9, “. . between 2011 and 2014 ... negative bias 2015", page13 line29-30, “. . period 2011-2015 ... during the 2016"

Interactive comment on Atmos. Meas. Tech. Discuss., doi:10.5194/amt-2019-46, 2019. 